\title{
Reaction of Sour Passion Fruit Lineages and Hybrids to Bacterial Spot Caused by Xanthomonas axononopodis pv. passiflorae Under Protected Cultivation and Field Conditions
}

\author{
Isadora Nogueira ${ }^{1}$, Anne Pinheiro Costa $^{1}$, José Ricardo Peixoto ${ }^{1} \&$ Michelle Souza Vilela ${ }^{1}$ \\ ${ }^{1}$ Faculty of Agronomy and Veterinary Medicine, University of Brasilia, Brasilia, Brazil \\ Correspondence: Isadora Nogueira, Faculty of Agronomy and Veterinary Medicine, University of Brasilia, \\ Brasilia, DF, Brazil. Tel: 55-61-996-828-910. E-mail: i.nogueiraa@gmail.com
}

Received: February 19, 2021

Accepted: May 6, $2021 \quad$ Online Published: August 15, 2021

doi:10.5539/jas.v13n9p112

URL: https://doi.org/10.5539/jas.v13n9p112

\begin{abstract}
Passion fruit is infected by many plant pathogens, including Xanthomonas axonopodis pv. passiflorae, causing bacterial spot disease. This disease has been impaired orchards all over Brazil, resulting in low yield and production. In addition, it results in premature death of plants in the field. This study aimed to analyze the reaction of sour passion fruit genotypes to bacterial spot in leaves under protected cultivation (Experiment 1) and the bacterial spot severity in fruits under field conditions (Experiment 2). Experiment 1 was carried out as a randomized block design (RBD) with subdivided plots, composed of 24 treatments (genotypes), three replications, five plants per plot, and five evaluation dates. Bacterial spot incidence and severity were evaluated using a 0 to 5 grading scale. Evaluations were carried out at a 7-day interval after disease symptoms first appeared. Genotypes differed for mean disease severity and incidence. The bacterial spot disease evaluations showed that genotypes MAR20\#46 P3 R4 X Rosa Claro R4 and MD 16 P3 X MAR20\#39 P1 R4 presented the lowest scores for the disease incidence in the leaves. S2L AP R1, MAR20\#19 ROXO R4 X ECRAM P3 R3, and MD 16 P3 X MAR20\#39 P1 R4 were the genotypes with the lowest disease severity scores. Experiment 2 consisted of a RBD with 24 treatments, three replications, and seven plants per plot. Severity assessments were monthly performed on five fruits per plot, totaling three evaluation dates. In this experiment, the percentage of total fruit area with necrotic lesions was measured using a 1 to 4 grading scale. Bacterial spot severity assessments in fruits identified interactions between genotypes and evaluation dates $(p \leq 0.05)$. The lineages S2L MAR 20\#15 R4 and S2L MAR20\#19 R2 showed the lowest mean severity scores of bacterial spot in fruit. The genotypes with the lowest mean scores were selected to continue the breeding program.
\end{abstract}

Keywords: bacterial spot, disease resistance, genetic breeding, Passiflora edulis Sims

\section{Introduction}

The passion fruit production was estimated at 593,429 tons during the 2018/2019 season in Brazil, with a cultivated area corresponding to 41,584 hectares (Brazilian Institute of Geography and Statistics [IBGE], 2019). The Brazilian mean yield is of 14.27 tons ha/year. However, the crop has the potential to reach 50 tons ha/year through the use of genetically improved cultivars and appropriate technologies in the production system (Faleiro \& Junqueira, 2011).

Passion fruit can be infected by various fungi, viruses, and bacteria, including Xanthomonas axonopodis pv. passiflorae, which causes bacterial spot disease. This disease may shorten the crop's productive cycle and cause the early death of plants. As a consequence, social and economic losses are observed since the curative control of diseases is costly and often not satisfactory (Miranda, 2004; Laranjeira, 2005). Since passion fruit presents considerable genetic variability, several breeding programs are focused on obtaining more productive and disease-resistant varieties (Costa et al., 2020).

The lack of productive and disease resistant genotypes contributes to the reduction of the passion fruit cultivated area. In its turn, passion fruit genetic breeding aims to obtain genotypes with higher performance regarding yield, production, fruit quality, and disease tolerance while meeting the market demands with reduced production costs and increased yield (Zaccheo, Aguiar, Stendel, Sera, \& Neves, 2012; Costa et al., 2018). 
Previous studies have already indicated the existence of genetic variability among genotypes from the passion fruit breeding program of the University of Brasilia. Therefore, the objective of this study was to evaluate genotypes of passion fruit regarding their resistance to bacterial spot disease under protected cultivation and field conditions.

\section{Method}

\subsection{Experimental Sites}

The experiments were performed under protected cultivation (Experiment 1), from February to March 2017, at the Experimental Biology Station (EBS) of the University of Brasilia (UnB); and under field conditions (Experiment 2), from March to May 2017, at the Água Limpa Farm (FAL) from UnB. Both EBS and FAL are located in Brasilia, $\mathrm{DF}$, Brazil $\left(16^{\circ} \mathrm{S}\right.$ and $48^{\circ} \mathrm{W}, 1010 \mathrm{~m}$ above sea level). The climate of the region is considered as $\mathrm{AW}$, according to the Köppen classification (Alvares, Stape, Sentelhas, Gonçalves, \& Sparovek, 2013), with rainy summer (October-April) and dry winter (May-September). The minimum and maximum temperatures registered during the studied period were 14 and $26{ }^{\circ} \mathrm{C}$, respectively. Minimum relative humidity of $62 \%$ and maximum relative humidity of $80 \%$ were recorded.

\subsection{Plant Material}

The genetic material evaluated were obtained from 24 superior genotypes belonging to the research program developed by Embrapa (Empresa Brasileira de Pesquisa Agropecuária-Brazilian Agricultural Research Corporation) and UnB (Costa et al., 2018). These genotypes were obtained by several recurrent selection cycles based on half-sib families, which were previously performed at the Água Limpa Farm (FAL) from UnB (Table 1). Seven out of the 24 materials tested were originated by self-fertilization (lineages) while 16 were originated by crosses of superior materials. One commercial cultivar was used as the control treatment.

Table 1. Passion fruit genotypes evaluated in the Federal District and their respective origins. Brasilia, DF, Brazil, 2017

\begin{tabular}{lll|lll}
\hline No. & Genotype & Origin & No. & Genotype & Origin \\
\hline 1 & Rosa Intenso P2 R4 & Lineage & 13 & MAR20\#21 R4 X FB200 R4 & $F_{1}$ hybrid \\
2 & AR2 R4 & Lineage & 14 & MD 16 P3 X MAR20\#39 P1 R4 & $F_{1}$ hybrid \\
3 & MSCA P1 R4 & Lineage & 15 & MAR20\#19 P1 R3 X MAR20\#2005 P3 R3 & $\mathrm{F}_{1}$ hybrid \\
4 & S2L62 & Lineage & 16 & Rosa Intenso P2 R4 X MSCA P1 R1 & $\mathrm{F}_{1}$ hybrid \\
5 & S2L MAR 20\#15 R4 & Lineage & 17 & EC3-0 X MAR20\#40 R3 & $\mathrm{F}_{1}$ hybrid \\
6 & S2L MAR20\#19 R2 & Lineage & 18 & MAR20\#24 X ECL7 P1 R4 & $\mathrm{F}_{1}$ hybrid \\
7 & S2L AP R1 & Lineage & 19 & MAR20\#100 R2 X MAR20\#21 R1 & $\mathrm{F}_{1}$ hybrid \\
8 & MAR20\#44 R4 x ECL7 P2 R4 & $\mathrm{F}_{1}$ hybrid & 20 & Rubi Gigante P1 R2 X MAR 20\#15 R2 & $\mathrm{F}_{1}$ hybrid \\
9 & MAR20\#46 P3 R4 X Rosa Claro R4 & $\mathrm{F}_{1}$ hybrid & 21 & MAR20\#24 P1 R2 X MSCA P1 R2 & $\mathrm{F}_{1}$ hybrid \\
10 & MAR20\#19 X MAR20\#21 P1 R4 & $\mathrm{F}_{1}$ hybrid & 22 & FB200 P1 R2 X MAR20\#2005 P1 R3 & $\mathrm{F}_{1}$ hybrid \\
11 & MAR20\#19 ROXO R4 X ECRAM P3 R3 & $\mathrm{F}_{1}$ hybrid & 23 & BRS Gigante Amarelo & Comercial cultivar \\
12 & MAR20\#24 R4 X MAR20\#40 & $\mathrm{F}_{1}$ hybrid & 24 & MAR20\#24 P1 R4 X Rosa Claro P2 R4 & $\mathrm{F}_{1}$ hybrid \\
\hline
\end{tabular}

Seedlings of each genotype were produced from seeds in 128-cell $(40 \mathrm{~mL} /$ cell $)$ extended polystyrene trays filled with vermiculite and bark of Pinus sp. (Vivatto Slim Plus ${ }^{\circledR}$; Technes Agrícola Ltda). At 30 days after seed germination, the seedlings were transplanted into 2-liter plastic bags containing a mixture of soil and fertilizer.

Plants were maintained under protected cultivation with daily irrigation and fertilization every two weeks with urea dissolved in water $(0.1 \mathrm{~g} /$ plant at each fertilization event) until experiment initiation (Experiment 1) or transplantation to the field (Experiment 2).

Plants destined to Experiment 2 were field transplanted at 90 days after sowing. The plants were arranged in the field at a spacing of $2.8 \mathrm{~m}$ (between-rows) by $3 \mathrm{~m}$ (within-rows), totaling 1,190 plants. The crop was managed using a trellis fence system comprised of 6 m-distant wooden stakes and two pieces of smooth wire $\left(n^{\circ} 12\right)$, at 1.6 and $2.2 \mathrm{~m}$ from the ground. Plants were grown as single-stem and tutored up to the top wire. Two lateral sprouts with opposite directions were left per wire. No chemical control of diseases or artificial pollination was performed to increase fruiting during the experiment evaluation period. 


\subsection{Disease Assessments}

\subsubsection{Experiment 1}

At 90 days after sowing, 15 plants of each material, each of them with about seven pairs of leaves, were inoculated with Xanthomonas axonopodis pv. passiflorae. Bacteria inoculation was performed using the UnB-1393 Xanthomonas axonopodis pv. passiflorae strain, which was obtained from the Bacteriology Laboratory at UnB. The UnB-1393 strain was multiplied using 523 culture media (Kado \& Heskett, 1970) at 28-30 ${ }^{\circ} \mathrm{C}$, for $72 \mathrm{~h}$ (Franco \& Takatsu, 2004). The bacterial suspension concentration $\left(\sim 1 \times 10^{6} \mathrm{CFU} / \mathrm{mL}\right)$ was adjusted on a spectrophotometer to an optical density of 0.145 at $550 \mathrm{~nm}$ wavelength, previously determined by the calibration curve (Costa et al., 2018).

The inoculation was performed with the aid of four needles fixed around the terminal part of a stick, forming a circle. At each perforation, the needles were simultaneously immersed in the bacterial suspension $\left(1 \times 10^{6}\right.$ $\mathrm{CFU} / \mathrm{mL}$ ). Three young leaves were inoculated with the bacteria. Each leaf was pierced once and simultaneously by the four needles, totaling four perforations per leaf (Costa et al., 2018). After inoculation, the plants were kept in a humid chamber for 72 hours.

Disease severity (\% total leaf area with necrotic lesions) and incidence (\% plants infected) were evaluated for five consecutive weeks after the onset of symptoms on plants. For disease severity assessment, a 0 to 5 scale used by Viana et al. (2014) and adapted by Costa et al. (2018) was used, as follows: 0 - no symptoms; $1-1$ to $10 \%$ of total leaf area with necrotic lesions; $2-11$ to $25 \%$ of total leaf area with necrotic lesions; $3-26$ to $50 \%$ of total leaf area with necrotic lesions; 4 -more than $50 \%$ of total leaf area with necrotic lesions; and 5-leaf drop. Based on the mean disease severity (DS) scores obtained from this scale, plants were classified as: resistant (R), $0 \leq \mathrm{DS}<1$; moderately resistant (MR), $1 \leq \mathrm{DS}<2$; moderately susceptible (MS), $2 \leq \mathrm{DS}<3$; susceptible (S), $3 \leq \mathrm{DS}<4$; and highly susceptible (HS), DS $\geq 4$ (Viana, Pires, Peixoto, Junqueira, \& Blum, 2014).

The area under the disease progress curve (AUDPC) was calculated using DS score data collected in the five evaluation dates (Campbell \& Madden, 1990).

\subsubsection{Experiment 2}

Fruits were harvested from the ground, at full physiological maturation stage. Disease severity was monthly evaluated in five fruits per plot, totaling three evaluation dates. The bacterial spot severity was calculated as the percentage of total fruit area with lesions. For severity assessments, a 1 to 4 scale (Junqueira, Anjos, Silva, Chaves, \& Gomes, 2003) was used, as follows: 1 -no symptoms; $2-1$ to $10.01 \%$ of total fruit area with lesions; $3-10.1$ to $30 \%$ of total fruit area with lesions; and $4-$ more than $30.01 \%$ of total fruit area with lesions. Based on the mean disease severity (DS) scores obtained from this scale, plants were classified as: resistant (R), $1 \leq \mathrm{DS}$ $\leq 1.5$; moderately resistant (MR), $1.51<\mathrm{DS} \leq 2.5$; susceptible (S), $2.51<\mathrm{DS} \leq 3.5$; and highly susceptible (HS), $3.51<\mathrm{DS} \leq 4$.

\subsection{Experimental Design and Data Analysis}

Experiment 1 consisted of a randomized block design (RBD) with subdivided parcels comprised of 24 treatments (genotypes), three repetitions, five plants per parcel, and five evaluation dates. Experiment 2 consisted of an RBD with 24 treatments, three repetitions, and seven plants per parcel.

Data were subject to analysis of variance using the F-test, and the means were grouped by the Scott-Knott's, at 5\% probability. Regression and linear correlation analyses were performed between the evaluated variables, based on the significance of their coefficients. Statistical analyses were performed using the SANEST software 5.6, Build 86. Correlation intensity was classified according to the magnitude of the values, as suggested by Carvalho et al. (2004): $\mathrm{r}=0$ (null); $0<|\mathrm{r}| \leq 0.30$ (weak); $0.30<|\mathrm{r}| \leq 0.60$ (medium); $0.60<|\mathrm{r}| \leq 0.90$ (strong); $0.90<|\mathrm{r}| \leq 1$ (very strong); and $|\mathbf{r}|=1$ (perfect).

Disease severity and incidence broad sense heritability $\left(h_{a}\right)$, genetic coefficient of variation (GCV), and genetic and environmental coefficient of variation ratio $(\mathrm{GCV} / \mathrm{ECV})$ were estimated using the GENES software (v. 1990.2017.37).

\section{Results and Discussion}

\subsection{Bacterial Spot Disease Evaluation Under Protected Cultivation}

Bacterial spot severity and incidence assessments identified interactions between genotypes and evaluation dates ( $\mathrm{p} \leq$ 0.05; Tables 2, 3, and 4). The hybrids MAR20\#46 P3 R4 X Rosa Claro R4 and MD 16 P3 X MAR20\#39 P1 R4 presented the lowest bacterial spot mean incidence scores in the leaves. Genotypes S2L AP R1, MAR20\#19 
ROXO R4 X ECRAM P3 R3, and MD 16 P3 X MAR20\#39 P1 R4 stood out for presenting the lowest bacterial spot mean severity scores, differing from the remaining genotypes, as shown in Table $2(\mathrm{p} \leq 0.05)$.

It is important to emphasize that the genotypes with the lowest bacterial spot mean incidence and severity scores differed from the commercial cultivar BRS Gigante Amarelo $(\mathrm{p} \leq 0.05)$, used in this study as control, which is classified as highly susceptible. Therefore, our findings represent progress in the passion fruit genetic breeding process towards the resistance to bacterial spot disease.

Table 2. Bacterial spot (Xanthomonas axonopodis pv. passiflorae) disease incidence (\%) and severity mean scores in leaves of 24 sour passion fruit genotypes (Passiflora edulis Sims), assessed in five evaluation dates, under protected cultivation. Brasilia, DF, Brazil, 2018

\begin{tabular}{lll}
\hline Genotypes & Incidence & Severity \\
\hline MAR20\#46 P3 R4 X Rosa Claro R4 & $88.25 \mathrm{a}$ & $2.36 \mathrm{~b}$ \\
MD 16 P3 X MAR20\#39 P1 R4 & $88.29 \mathrm{a}$ & $2.22 \mathrm{a}$ \\
S2L AP R1 & $91.55 \mathrm{~b}$ & $1.96 \mathrm{a}$ \\
MAR20\#19 X MAR20\#21 P1 R4 & $93.11 \mathrm{~b}$ & $2.37 \mathrm{~b}$ \\
MAR20\#24 P1 R4 X Rosa Claro P2 & $93.33 \mathrm{~b}$ & $2.67 \mathrm{~b}$ \\
MAR20\#19 ROXO R4 X ECRAM P3 R3 & $93.77 \mathrm{~b}$ & $2.06 \mathrm{a}$ \\
FB200 P1 R2 X MAR20\#2005 P1 R3 & $94.10 \mathrm{~b}$ & $3.01 \mathrm{c}$ \\
MAR20\#19 P1 R3 X MAR20\#2005 P3 & $94.20 \mathrm{~b}$ & $3.25 \mathrm{c}$ \\
MAR20\#21 R4 X FB200 R4 & $94.33 \mathrm{~b}$ & $2.87 \mathrm{c}$ \\
Rubi Gigante P1 R2 X MAR 20\#15 & $94.44 \mathrm{~b}$ & $2.72 \mathrm{~b}$ \\
MAR20\#100 R2 X MAR20\#21 R1 & $94.83 \mathrm{~b}$ & $2.46 \mathrm{~b}$ \\
Rosa Intenso P2 R4 & $95.12 \mathrm{~b}$ & $2.62 \mathrm{~b}$ \\
Rosa Intenso P2 R4 X MSCA P1 R & $95.12 \mathrm{~b}$ & $3.45 \mathrm{~d}$ \\
MAR20\#44 R4 x ECL7 P2 R4 & $96.66 \mathrm{c}$ & $3.10 \mathrm{c}$ \\
AR2 R4 & $96.88 \mathrm{c}$ & $3.24 \mathrm{c}$ \\
MSCA P1 R4 & $97.06 \mathrm{c}$ & $3.71 \mathrm{~d}$ \\
S2L62 & $97.30 \mathrm{c}$ & $3.56 \mathrm{~d}$ \\
MAR20\#24 R4 X MAR20\#40 & $97.46 \mathrm{c}$ & $3.04 \mathrm{c}$ \\
S2L MAR 20\#15 R4 & $98.44 \mathrm{c}$ & $3.42 \mathrm{~d}$ \\
MAR20\#24 P1 R2 X MSCA P1 R2 & $98.51 \mathrm{c}$ & $3.11 \mathrm{c}$ \\
S2L MAR20\#19 R2 & $98.78 \mathrm{c}$ & $3.09 \mathrm{c}$ \\
EC3-0 X MAR20\#40 R3 & $98.81 \mathrm{c}$ & $3.16 \mathrm{c}$ \\
BRS Gigante Amarelo & $98.99 \mathrm{c}$ & $3.73 \mathrm{~d}$ \\
MAR20\#24 X ECL7 P1 R4 & $99.55 \mathrm{c}$ & $3.30 \mathrm{c}$ \\
\hline
\end{tabular}

Note. Means followed by different letters in the column differ by the Scott-Knott's test at 5\% probability.

Similar to the results here presented, Fuhrmann et al. (2014) classified BRS Gigante Amarelo as the most susceptible cultivar to bacterial spot in a study assessing the resistance degree of 36 passion fruit clones to three isolates of Xanthomonas axonopodis pv. passiflorae.

Differences among genotypes were verified for disease incidence and severity scores at the evaluations 14,21 , 28, and 36 days after inoculation ( $\mathrm{p} \leq 0.05$; Tables 3 and 4). At evaluation 1 , the disease was at its initial stage, and no differences were identified.

MAR20\#46 P3 R4 X Rosa Claro R4 and MD 16 P3 X MAR20\#39 P1 R4 were the only hybrids not showing differences in the disease incidence among the five evaluation dates (Table 3). The hybrids MAR20\#19 ROXO R4 X ECRAM P3 R3, MAR20\#19 X MAR20\#21 P1 R4, and MAR20\#46 P3 R4 X Rosa Claro R4, as well as the lineage S2L AP R1 did not show differences in the disease severity among the five evaluation dates (Table 4). 
Table 3. Interaction between evaluation dates and genotypes in the assessment of the bacterial spot (Xanthomonas axonopodis pv. passiflorae) disease incidence (\%) in leaves of 24 sour passion fruit genotypes (Passiflora edulis Sims), under protected cultivation. Brasilia, DF, Brazil, 2017

\begin{tabular}{|c|c|c|c|c|c|c|}
\hline \multirow{2}{*}{ Genotype } & \multicolumn{5}{|c|}{ Incidence at each evaluation date } & \multirow{2}{*}{ DI } \\
\hline & 7 DAI & 14 DAI & 21 DAI & 28 DAI & 35 DAI & \\
\hline AR2 R4 & $84.4 \mathrm{aB}$ & $100 \mathrm{bB}$ & $100 \mathrm{bA}$ & $100 \mathrm{bA}$ & $100 \mathrm{bA}$ & $96.88 \mathrm{c}$ \\
\hline EC3-0 X MAR20\#40 R3 & $94.01 \mathrm{aC}$ & $100 \mathrm{aB}$ & $100 \mathrm{aA}$ & $100 \mathrm{aA}$ & $100 \mathrm{aA}$ & $98.81 \mathrm{c}$ \\
\hline FB200 P1 R2 X MAR20\#2005 P1 R3 & $79.62 \mathrm{aB}$ & $90 \mathrm{bA}$ & $100 \mathrm{bA}$ & $100 \mathrm{bA}$ & $100 \mathrm{bA}$ & $94.10 \mathrm{~b}$ \\
\hline BRS Gigante Amarelo & $94.99 \mathrm{aC}$ & $100 \mathrm{aB}$ & $100 \mathrm{aA}$ & $100 \mathrm{aA}$ & $100 \mathrm{aA}$ & $98.99 \mathrm{c}$ \\
\hline MAR20\#100 R2 X MAR20\#21 R1 & $83.72 \mathrm{aB}$ & $95.23 \mathrm{bB}$ & $95.23 \mathrm{bA}$ & $100 \mathrm{bA}$ & $100 \mathrm{bA}$ & $94.83 \mathrm{~b}$ \\
\hline MAR20\#19 P1 R3 X MAR20\#2005 P3 & $79.62 \mathrm{aB}$ & $94.18 \mathrm{bB}$ & $97.23 \mathrm{bA}$ & $100 \mathrm{bA}$ & $100 \mathrm{bA}$ & $94.20 \mathrm{~b}$ \\
\hline MAR20\#19 ROXO R4 X ECRAM P3 R3 & $82.22 \mathrm{aB}$ & $93.33 \mathrm{bB}$ & $93.33 \mathrm{bA}$ & $100 \mathrm{bA}$ & $100 \mathrm{bA}$ & $93.77 \mathrm{~b}$ \\
\hline MAR20\#19 X MAR20\#21 P1 R4 & $82.79 \mathrm{aB}$ & $90.20 \mathrm{aA}$ & $92.59 \mathrm{aA}$ & $100 \mathrm{bA}$ & $100 \mathrm{bA}$ & $93.11 \mathrm{~b}$ \\
\hline MAR20\#21 R4 X FB200 R4 & $82.77 \mathrm{aB}$ & $94.44 \mathrm{aB}$ & $94.44 \mathrm{aA}$ & $100 \mathrm{bA}$ & $100 \mathrm{bA}$ & $94.33 \mathrm{~b}$ \\
\hline MAR20\#24 P1 R2 X MSCA P1 R2 & $96.29 \mathrm{aC}$ & $100 \mathrm{aB}$ & $100 \mathrm{aA}$ & $100 \mathrm{aA}$ & $100 \mathrm{aA}$ & $98.51 \mathrm{c}$ \\
\hline MAR20\#24 P1 R4 X Rosa Claro P2 & $75 \mathrm{aB}$ & $97.22 \mathrm{bB}$ & $97.22 \mathrm{bA}$ & $97.22 \mathrm{bA}$ & $100 \mathrm{bA}$ & $93.33 \mathrm{~b}$ \\
\hline MAR20\#24 R4 X MAR20\#40 & $87.3 \mathrm{aC}$ & $100 \mathrm{bB}$ & $100 \mathrm{bA}$ & $100 \mathrm{bA}$ & $100 \mathrm{bA}$ & $97.46 \mathrm{c}$ \\
\hline MAR20\#24 X ECL7 P1 R4 & $97.77 \mathrm{aC}$ & $100 \mathrm{bB}$ & $100 \mathrm{bA}$ & $100 \mathrm{bA}$ & $100 \mathrm{bA}$ & $99.55 \mathrm{c}$ \\
\hline MAR20\#44 R4 x ECL7 P2 R4 & $83.33 \mathrm{aB}$ & $100 \mathrm{bB}$ & $100 \mathrm{bA}$ & $100 \mathrm{bA}$ & $100 \mathrm{bA}$ & $96.66 \mathrm{c}$ \\
\hline MAR20\#46 P3 R4 X Rosa Claro R4 & $65.92 \mathrm{aA}$ & $78.38 \mathrm{bA}$ & $96.96 \mathrm{cA}$ & $100 \mathrm{cA}$ & $100 \mathrm{cA}$ & $88.25 \mathrm{a}$ \\
\hline MD 16 P3 X MAR20\#39 P1 R4 & $63.17 \mathrm{aA}$ & $87.22 \mathrm{bA}$ & $95.55 \mathrm{bA}$ & $95.55 \mathrm{bA}$ & $100 \mathrm{bA}$ & $88.29 \mathrm{a}$ \\
\hline MSCA P1 R4 & $91.15 \mathrm{aC}$ & $91.18 \mathrm{aB}$ & $100 \mathrm{aA}$ & $100 \mathrm{aA}$ & $100 \mathrm{aA}$ & $97.06 \mathrm{c}$ \\
\hline Rosa Intenso P2 R4 & $79.99 \mathrm{aB}$ & $95.55 \mathrm{bB}$ & $100 \mathrm{bA}$ & $100 \mathrm{bA}$ & $100 \mathrm{bA}$ & $95.12 \mathrm{~b}$ \\
\hline Rosa Intenso P2 R4 X MSCA P1 R & $78.02 \mathrm{aB}$ & $97.61 \mathrm{bB}$ & $100 \mathrm{bA}$ & $100 \mathrm{bA}$ & $100 \mathrm{bA}$ & $95.12 \mathrm{~b}$ \\
\hline Rubi Gigante P1 R2 X MAR 20\#15 & $80.55 \mathrm{aB}$ & $95.83 \mathrm{bB}$ & $95.83 \mathrm{bA}$ & $100 \mathrm{bA}$ & $100 \mathrm{bA}$ & $94.44 \mathrm{~b}$ \\
\hline S2L AP R1 & $75.12 \mathrm{aC}$ & $85.04 \mathrm{aA}$ & $97.61 \mathrm{bA}$ & $100 \mathrm{bA}$ & $100 \mathrm{bA}$ & $91.55 \mathrm{~b}$ \\
\hline S2L MAR 20\#15 R4 & $92.22 \mathrm{aC}$ & $100 \mathrm{aB}$ & $100 \mathrm{aA}$ & $100 \mathrm{aA}$ & $100 \mathrm{aA}$ & $98.44 \mathrm{c}$ \\
\hline S2L MAR20\#19 R2 & $93.93 \mathrm{aC}$ & $100 \mathrm{aB}$ & $100 \mathrm{aA}$ & $100 \mathrm{aA}$ & $100 \mathrm{aA}$ & $98.78 \mathrm{c}$ \\
\hline S2L62 & $90.23 \mathrm{aC}$ & $96.29 \mathrm{aB}$ & $100 \mathrm{aA}$ & $100 \mathrm{aA}$ & $100 \mathrm{aA}$ & $97.30 \mathrm{c}$ \\
\hline
\end{tabular}

Note. DAI = Days after inoculation; DI = Mean incidence scores of five evaluations. Means followed by different lowercase letters within columns and uppercase letters within rows differ by the Scott-Knott's test at 5\% probability.

At the second evaluation date (14 days after inoculation) $37.5 \%$ of the genotypes already had $100 \%$ of plants with symptoms of the disease. At 28 days after inoculation, only two genotypes did not reach $100 \%$ incidence (Table 3). These genotypes, two F1 hybrids (MAR20\#24 P1 R4 X Rosa Claro P2 and MD 16 P3 X MAR20\#39 $\mathrm{P} 1 \mathrm{R} 4)$, will be selected for further studies in the passion fruit genetic breeding process aiming at screening for resistance to the bacterial spot. 
Table 4. Interaction between evaluation dates and genotypes in the assessment of the bacterial spot (Xanthomonas axonopodis pv. passiflorae) disease severity in leaves of 24 sour passion fruit genotypes (Passiflora edulis Sims), under protected cultivation. Brasilia, DF, Brazil, 2017

\begin{tabular}{|c|c|c|c|c|c|c|c|}
\hline \multirow{2}{*}{ Genotype } & \multicolumn{5}{|c|}{ Severity at each evaluation date } & \multirow{2}{*}{ DS } & \multirow{2}{*}{$\mathbf{R R}$} \\
\hline & 7 DAI & 14 DAI & 21 DAI & 28 DAI & 35 DAI & & \\
\hline AR2 R4 & $0.91 \mathrm{aA}$ & $2.76 \mathrm{bB}$ & $3.79 \mathrm{cB}$ & $4.17 \mathrm{cB}$ & $4.60 \mathrm{cB}$ & $3.24 \mathrm{c}$ & $\mathrm{S}$ \\
\hline EC3-0 X MAR20\#40 R3 & $0.91 \mathrm{aA}$ & $1.87 \mathrm{aA}$ & $3.68 \mathrm{bB}$ & $4.51 \mathrm{bB}$ & $4.85 \mathrm{bB}$ & $3.16 \mathrm{c}$ & S \\
\hline FB200 P1 R2 X MAR20\#2005 P1 R3 & $0.79 \mathrm{aA}$ & $2.16 \mathrm{bA}$ & $3.47 \mathrm{cB}$ & $4.14 \mathrm{cB}$ & $4.47 \mathrm{cB}$ & $3.01 \mathrm{c}$ & HS \\
\hline BRS Gigante Amarelo & $1.06 \mathrm{aA}$ & $3.07 \mathrm{bB}$ & $4.72 \mathrm{cB}$ & $4.89 \mathrm{cB}$ & $4.91 \mathrm{cB}$ & $3.73 \mathrm{~d}$ & HS \\
\hline MAR20\#100 R2 X MAR20\#21 R1 & $0.89 \mathrm{aA}$ & $1.7 \mathrm{aA}$ & $2.77 \mathrm{bA}$ & $3.36 \mathrm{bA}$ & $3.56 \mathrm{bB}$ & $2.46 \mathrm{~b}$ & MS \\
\hline MAR20\#19 P1 R3 X MAR20\#2005 P3 & $0.91 \mathrm{aA}$ & $2.67 \mathrm{bB}$ & $3.78 \mathrm{cB}$ & $4.28 \mathrm{cB}$ & $4.61 \mathrm{cB}$ & $3.25 \mathrm{c}$ & HS \\
\hline MAR20\#19 ROXO R4 X ECRAM P3 R3 & $1.07 \mathrm{aA}$ & $1.99 \mathrm{bA}$ & $2.09 \mathrm{bA}$ & $2.29 \mathrm{bA}$ & $2.86 \mathrm{bA}$ & $2.06 \mathrm{a}$ & MS \\
\hline MAR20\#19 X MAR20\#21 P1 R4 & $1.09 \mathrm{aA}$ & $1.68 \mathrm{aA}$ & $2.78 \mathrm{bA}$ & $2.84 \mathrm{bA}$ & $3.45 \mathrm{bA}$ & $2.37 \mathrm{~b}$ & MS \\
\hline MAR20\#21 R4 X FB200 R4 & $0.82 \mathrm{aA}$ & $2.25 \mathrm{bA}$ & $3.55 \mathrm{cB}$ & $3.79 \mathrm{cB}$ & $3.95 \mathrm{cA}$ & $2.87 \mathrm{c}$ & $\mathrm{S}$ \\
\hline MAR20\#24 P1 R2 X MSCA P1 R2 & $1.02 \mathrm{aA}$ & $2.16 \mathrm{bA}$ & $3.58 \mathrm{cB}$ & $4.38 \mathrm{cB}$ & $4.44 \mathrm{cB}$ & $3.11 \mathrm{c}$ & $\mathrm{S}$ \\
\hline MAR20\#24 P1 R4 X Rosa Claro P2 & $0.77 \mathrm{aA}$ & $1.62 \mathrm{aA}$ & $2.74 \mathrm{bA}$ & $4.04 \mathrm{cB}$ & $4.20 \mathrm{cB}$ & $2.67 \mathrm{~b}$ & MS \\
\hline MAR20\#24 R4 X MAR20\#40 & $0.92 \mathrm{aA}$ & $2.91 \mathrm{bB}$ & $3.29 \mathrm{bB}$ & $4.04 \mathrm{cB}$ & $4.07 \mathrm{cB}$ & $3.04 \mathrm{c}$ & $\mathrm{S}$ \\
\hline MAR20\#24 X ECL7 P1 R4 & $1.00 \mathrm{aA}$ & $2.47 \mathrm{bB}$ & $3.89 \mathrm{cB}$ & $4.36 \mathrm{cB}$ & $4.80 \mathrm{cB}$ & $3.30 \mathrm{c}$ & $\mathrm{S}$ \\
\hline MAR20\#44 R4 x ECL7 P2 R4 & $0.93 \mathrm{aA}$ & $2.58 \mathrm{bB}$ & $3.34 \mathrm{cB}$ & $4.11 \mathrm{cB}$ & $4.53 \mathrm{cB}$ & $3.10 \mathrm{c}$ & HS \\
\hline MAR20\#46 P3 R4 X Rosa Claro R4 & $0.75 \mathrm{aA}$ & $1.28 \mathrm{aA}$ & $2.72 \mathrm{bA}$ & $3.52 \mathrm{bA}$ & $3.55 \mathrm{bA}$ & $2.36 \mathrm{~b}$ & MS \\
\hline MD 16 P3 X MAR20\#39 P1 R4 & $0.65 \mathrm{aA}$ & $1.53 \mathrm{aA}$ & $2.28 \mathrm{bA}$ & $2.85 \mathrm{bA}$ & $3.79 \mathrm{cB}$ & $2.22 \mathrm{a}$ & MS \\
\hline MSCA P1 R4 & $1.24 \mathrm{aA}$ & $3.36 \mathrm{bA}$ & $4.42 \mathrm{cB}$ & $4.68 \mathrm{cB}$ & $4.86 \mathrm{cB}$ & $3.71 \mathrm{~d}$ & HS \\
\hline Rosa Intenso P2 R4 & $0.96 \mathrm{aA}$ & $1.79 \mathrm{aB}$ & $3.27 \mathrm{bB}$ & $3.40 \mathrm{bA}$ & $3.86 \mathrm{cA}$ & $2.62 \mathrm{~b}$ & $\mathrm{~S}$ \\
\hline Rosa Intenso P2 R4 X MSCA P1 R & $0.78 \mathrm{aA}$ & $2.70 \mathrm{bB}$ & $4.17 \mathrm{cB}$ & $4.67 \mathrm{cB}$ & $4.92 \mathrm{cB}$ & $3.45 \mathrm{~d}$ & $\mathrm{~S}$ \\
\hline Rubi Gigante P1 R2 X MAR 20\#15 & $0.89 \mathrm{aA}$ & $2.05 \mathrm{bA}$ & $2.91 \mathrm{cA}$ & $3.50 \mathrm{cA}$ & $4.26 \mathrm{~dB}$ & $2.72 \mathrm{~b}$ & $\mathrm{~S}$ \\
\hline S2L AP R1 & $0.74 \mathrm{aA}$ & $1.18 \mathrm{aA}$ & $2.22 \mathrm{bA}$ & $2.81 \mathrm{bA}$ & $2.88 \mathrm{bA}$ & $1.96 \mathrm{a}$ & MS \\
\hline S2L MAR 20\#15 R4 & $1.06 \mathrm{aA}$ & $3.18 \mathrm{bB}$ & $4.15 \mathrm{cB}$ & $4.29 \mathrm{cB}$ & $4.44 \mathrm{cB}$ & $3.42 \mathrm{~d}$ & $\mathrm{~S}$ \\
\hline S2L MAR20\#19 R2 & $0.93 \mathrm{aA}$ & $2.24 \mathrm{bA}$ & $3.61 \mathrm{cB}$ & $4.25 \mathrm{cB}$ & $4.44 \mathrm{cB}$ & $3.09 \mathrm{c}$ & $\mathrm{S}$ \\
\hline S2L62 & $1.06 \mathrm{aA}$ & $3.36 \mathrm{bB}$ & $4.25 \mathrm{cB}$ & $4.47 \mathrm{cB}$ & $4.67 \mathrm{cB}$ & $3.56 \mathrm{~d}$ & HS \\
\hline
\end{tabular}

Note. DAI $=$ Days after inoculation; $\mathrm{DS}=$ Mean severity scores of five evaluations; RR $=$ Resistance reaction. Means followed by different lowercase letters within columns and uppercase letters within rows differ by the Scott-Knott's test at 5\% probability.

Differences among genotypes were not observed for disease severity only at the first evaluation date. Despite the lack of difference among genotypes, it is important to emphasize that MD 16 P3 X MAR20\#39 P1 R4 (0.65) presented a severity score of 39\% lower than BRS Gigante Amarelo cultivar (1.06) (Table 4).

The hybrids MAR20\#19 ROXO R4 X ECRAM P3 R3, MAR20\#19 X MAR20\#21 P1 R4, and MAR20\#46 P3 R4 X Rosa Claro R4, as well as the lineage S2L AP R1 did not show differences in disease severity among the five evaluation dates. Along with MAR20\#100 R2 X MAR20\#21 R1, MAR20\#24 P1 R4 X Rosa P2, and MD 16 P3 X MAR20\#39 P1 R4 were classified as MS and were selected for following up with the breeding program on disease resistance (Table 4).

According to the mean disease severity, $29 \%$ of the genotypes were classified as MS, $46 \%$ as susceptible, and $25 \%$ highly susceptible. These results differ from those reported by Costa et al. (2018), who tested 11 genotypes in a greenhouse and obtained a lower percentage of MR genotypes.

Furthermore, Costa et al. (2018), and Costa, Nogueira, Peixoto, and Blum (2020) recorded the lowest severity scores for the cultivar BRS Gigante Amarelo in studies performed with different varieties. Unlikely, in the present study, BRS Gigante Amarelo had the highest severity scores, assuming greater resistance gain when comparing hybrids and selected lineages with varieties.

Our results also indicated differences among genotypes regarding their resistance to bacterial spot when considering the AUDPC (Table 5). 
Table 5. Area under the disease progress curve (AUPDC) in sour passion fruit genotypes (Passiflora edulis Sims) calculated using bacterial spot (Xanthomonas axonopodis pv. passiflorae) severity score data collected in five evaluation dates, under protected cultivation. Brasilia, DF, Brazil, 2018

\begin{tabular}{ll}
\hline Genotype & AUPDC \\
\hline S2L AP R1 & $56.23 \mathrm{a}$ \\
MAR20\#19 ROXO R4 X ECRAM P3 R3 & $58.49 \mathrm{a}$ \\
MD 16 P3 X MAR20\#39 P1 R4 & $62.21 \mathrm{a}$ \\
MAR20\#19 X MAR20\#21 P1 R4 & $67.14 \mathrm{a}$ \\
MAR20\#46 P3 R4 X Rosa Claro R4 & $67.84 \mathrm{a}$ \\
MAR20\#100 R2 X MAR20\#21 R1 & $70.52 \mathrm{a}$ \\
Rosa Intenso P2 R4 & $75.53 \mathrm{a}$ \\
MAR20\#24 P1 R4 X Rosa Claro P2 & $76.26 \mathrm{a}$ \\
Rubi Gigante P1 R2 X MAR 20\#15 & $77.32 \mathrm{a}$ \\
MAR20\#21 R4 X FB200 R4 & $83.88 \mathrm{~b}$ \\
FB200 P1 R2 X MAR20\#2005 P1 R3 & $86.95 \mathrm{~b}$ \\
MAR20\#24 R4 X MAR20\#40 & $89.20 \mathrm{~b}$ \\
S2L MAR20\#19 R2 & $89.56 \mathrm{~b}$ \\
MAR20\#24 P1 R2 X MSCA P1 R2 & $90.05 \mathrm{~b}$ \\
EC3-0 X MAR20\#40 R3 & $90.61 \mathrm{~b}$ \\
MAR20\#44 R4 x ECL7 P2 R4 & $91.03 \mathrm{~b}$ \\
AR2 R4 & $94.44 \mathrm{~b}$ \\
MAR20\#19 P1 R3 X MAR20\#2005 P3 & $94.57 \mathrm{~b}$ \\
MAR20\#24 X ECL7 P1 R4 & $95.38 \mathrm{~b}$ \\
S2L MAR 20\#15 R4 & $100.70 \mathrm{~b}$ \\
Rosa Intenso P2 R4 X MSCA P1 R & $100.84 \mathrm{~b}$ \\
S2L62 & $104.75 \mathrm{~b}$ \\
MSCA P1 R4 & $108.62 \mathrm{~b}$ \\
Gigante Amarelo Tropical & $109.85 \mathrm{~b}$ \\
\hline
\end{tabular}

Note. Means followed by different letters in the column differ by the Scott-Knott's test at 5\% probability.

The different results mentioned can be explained due to the genetic variability of the material obtained by seed, which is in the segregation process. Other disparities may also be explained by pathogen variability, through the use of different bacteria strain, isolate aggressiveness, inoculum concentration, and the inoculation method (Gonçalves, 2011).

The AUPDC manages to represent the epidemic as a whole since it considers the stress that the crop experienced during various stages of development (Bergamin Filho, Lopes, Amorim, Godoy, \& Berger, 1995).

The variation in the incidence index (\%) and the severity score of the disease with time was estimated by quadratic regression. It was found that the disease reached its peak before the end of the severity assessments. As far as incidence is concerned, its peak occurred between days 21 and 35 after inoculation for all genotypes.

Medium heritability values were observed for bacterial spot severity $(65.82 \%)$. Heritability measures the degree of correlation between phenotypic and genetic values. High heritability values indicate that simple selection methods, such as mass selection, can lead to considerable gains, considering the low influence of the environment over the trait (Falconer, 1987).

The genetic coefficient of variation was $18.4 \%$ for disease severity, indicating low genetic progress with selection. Additionally, the environmental coefficient of variation 18,4 pointed to considerable environmental influence on the trait. The value of the $\mathrm{CVg} / \mathrm{CVe}$ ratio was lower than 1 , indicating that simple selection methods would possibly not be suitable for the development resistant genotypes to bacterial spot (Rocha, 2014).

\subsection{Bacterial Spot Disease Evaluation in Fruits}

Different results from those recorded under protected cultivation were found for the same 24 genotypes when they were tested in the field. The field evaluation confirmed the results observed under protected cultivation regarding the resistance level of the genotypes under natural climatic conditions and natural inoculum pressure. 
Bacterial spot severity assessments in fruits identified interactions between genotypes and evaluation dates and differences among genotypes were observed $(\mathrm{p} \leq 0.05)$.

As a result, differences among genotypes were observed in the second evaluation date (Table 6). Differences among evaluation dates were also recorded for Rubi Gigante P1 R2 X MAR 20\#15, which showed the highest severity score at the second evaluation. Similarly, Junqueira et al. (2003) also reported a moderate resistance degree and $10 \%$ to $30 \%$ of the fruit surface covered with lesions in all of the 11 cultivars tested, although no differences among genotypes were recorded.

Table 6. Interaction between evaluation dates and genotypes in the assessment of the bacterial spot (Xanthomonas axonopodis pv. passiflorae) disease severity in fruits of 24 sour passion fruit genotypes (Passiflora edulis Sims). Brasilia, DF, Brazil, 2017

\begin{tabular}{|c|c|c|c|c|c|}
\hline Genotype & E1 & $\mathbf{E 2}$ & $\mathbf{E 3}$ & DS & $\mathbf{R R}$ \\
\hline AR2 R4 & $1.80 \mathrm{aA}$ & $1.60 \mathrm{aA}$ & $1.65 \mathrm{aA}$ & $1.68 \mathrm{a}$ & MR \\
\hline EC3-0 X MAR20\#40 R3 & $1.75 \mathrm{aA}$ & $1.80 \mathrm{aA}$ & $1.80 \mathrm{aA}$ & $1.78 \mathrm{a}$ & MR \\
\hline FB200 P1 R2 X MAR20\#2005 P1 R3 & $1.66 \mathrm{aA}$ & $2.06 \mathrm{bA}$ & $2.13 \mathrm{aA}$ & $1.95 \mathrm{a}$ & MR \\
\hline Gigante Amarelo Tropical & $1.93 \mathrm{aA}$ & $2.08 \mathrm{bA}$ & $2.00 \mathrm{aA}$ & $2.00 \mathrm{a}$ & MR \\
\hline MAR20\#100 R2 X MAR20\#21 R1 & $1.60 \mathrm{aA}$ & $1.86 \mathrm{aA}$ & $1.56 \mathrm{aA}$ & $1.67 \mathrm{a}$ & MR \\
\hline MAR20\#19 P1 R3 X MAR20\#2005 P3 & $1.83 \mathrm{aA}$ & $1.86 \mathrm{aA}$ & $2.00 \mathrm{aA}$ & $1.90 \mathrm{a}$ & MR \\
\hline MAR20\#19 ROXO R4 X ECRAM P3 R3 & $2.00 \mathrm{aA}$ & $1.80 \mathrm{aA}$ & $2.20 \mathrm{aA}$ & $2.00 \mathrm{a}$ & MR \\
\hline MAR20\#19 X MAR20\#21 P1 R4 & $1.83 \mathrm{aA}$ & $1.56 \mathrm{aA}$ & $1.86 \mathrm{aA}$ & $1.75 \mathrm{a}$ & MR \\
\hline MAR20\#21 R4 X FB200 R4 & $2.00 \mathrm{aA}$ & $1.73 \mathrm{aA}$ & $1.83 \mathrm{aA}$ & $1.85 \mathrm{a}$ & MR \\
\hline MAR20\#24 P1 R2 X MSCA P1 R2 & $1.80 \mathrm{aA}$ & $1.93 \mathrm{aA}$ & $1.83 \mathrm{aA}$ & $1.85 \mathrm{a}$ & MR \\
\hline MAR20\#24 P1 R4 X Rosa Claro P2 & $1.83 \mathrm{aA}$ & $2.33 \mathrm{bA}$ & $2.06 \mathrm{aA}$ & $2.07 \mathrm{a}$ & MR \\
\hline MAR20\#24 R4 X MAR20\#40 & $1.80 \mathrm{aA}$ & $2.13 \mathrm{bA}$ & $1.96 \mathrm{aA}$ & $1.96 \mathrm{a}$ & MR \\
\hline MAR20\#24 X ECL7 P1 R4 & $1.66 \mathrm{aA}$ & $1.73 \mathrm{aA}$ & $1.50 \mathrm{aA}$ & $1.63 \mathrm{a}$ & MR \\
\hline MAR20\#44 R4 x ECL7 P2 R4 & $2.18 \mathrm{aA}$ & $2.20 \mathrm{bA}$ & $1.88 \mathrm{aA}$ & $2.09 \mathrm{a}$ & MR \\
\hline MAR20\#46 P3 R4 X Rosa Claro R4 & $1.96 \mathrm{aA}$ & $1.43 \mathrm{aA}$ & $1.80 \mathrm{aA}$ & $1.73 \mathrm{a}$ & MR \\
\hline MD 16 P3 X MAR20\#39 P1 R4 & $1.86 \mathrm{aA}$ & $1.76 \mathrm{aA}$ & $1.83 \mathrm{aA}$ & $1.82 \mathrm{a}$ & MR \\
\hline MSCA P1 R4 & $1.90 \mathrm{aA}$ & $2.00 \mathrm{bA}$ & $2.00 \mathrm{aA}$ & $1.97 \mathrm{a}$ & MR \\
\hline Rosa Intenso P2 R4 & $1.68 \mathrm{aA}$ & $1.70 \mathrm{aA}$ & $1.93 \mathrm{aA}$ & $1.77 \mathrm{a}$ & MR \\
\hline Rosa Intenso P2 R4 X MSCA P1 R & $1.53 \mathrm{aA}$ & $1.76 \mathrm{aA}$ & $1.83 \mathrm{aA}$ & $1.71 \mathrm{a}$ & MR \\
\hline Rubi Gigante P1 R2 X MAR 20\#15 & $2.00 \mathrm{aA}$ & $2.56 \mathrm{bB}$ & $1.68 \mathrm{aA}$ & $2.08 \mathrm{a}$ & MR \\
\hline S2L AP R1 & $2.13 \mathrm{aA}$ & $1.80 \mathrm{aA}$ & $2.06 \mathrm{aA}$ & $2.00 \mathrm{a}$ & MR \\
\hline S2L MAR 20\#15 R4 & $1.23 \mathrm{aA}$ & $1.60 \mathrm{aA}$ & $1.80 \mathrm{aA}$ & $1.54 \mathrm{a}$ & MR \\
\hline S2L MAR20\#19 R2 & $1.61 \mathrm{aA}$ & $1.33 \mathrm{aA}$ & $1.73 \mathrm{aA}$ & $1.56 \mathrm{a}$ & MR \\
\hline S2L62 & $1.66 \mathrm{aA}$ & $1.60 \mathrm{aA}$ & $1.63 \mathrm{aA}$ & $1.63 \mathrm{a}$ & MR \\
\hline
\end{tabular}

Note. $\mathrm{E}=$ Evaluation; $\mathrm{DS}=$ Mean severity scores of three evaluations; $\mathrm{RR}=$ Resistance reaction. Means followed by different lowercase letters within columns and uppercase letters within rows differ by the Scott-Knott's test at 5\% probability.

The bacterial spot severity in fruits is affected by external factors, such as climatic conditions (Junqueira, Sussel, Junqueira, Zacaroni, \& Braga, 2016). The disease assessments were carried out in the months of March, April, and May, which differed from each other regarding the rainfall volume and relative humidity (Brazilian National Institute of Meteorology [INMET], 2019).

All genotypes were classified as MR. However, S2L MAR 20\#15 R4 and S2L MAR20\#19 R2 stood out as the lineages with the lowest disease mean severity scores in fruits. These findings differ from those recorded under protected cultivation, in which these lineages were classified as S. Therefore, this study emphasizes the importance of developing field trials and illustrates how much the susceptibility of particular plant material can interfere with its final product, the fruit. 
The variability to disease resistance sour passion fruit is low. As a consequence, any difference between and within genotypes under study must be taken into account, since it may be useful in the resistance selection process to bacterial spot (Junqueira et al. 2003; Fuhrmann et al., 2014; Costa et al., 2018).

\section{Conclusion}

Genotypes MAR20\#46 P3 R4 X Rosa Claro R4 and MD 16 P3 X MAR20\#39 P1 R4 presented the lowest bacterial spot incidence scores in the leaves. S2L AP R1, MAR20\#19 ROXO R4 X ECRAM P3 R3, and MD 16 P3 X MAR20\#39 P1 R4 were the genotypes with the lowest severity scores.

Low heritability values and GCV/ECV ratio were observed for the analyzed variables.

The lineages S2L MAR 20\#15 R4 and S2L MAR20\#19 R2 showed the lowest mean severity scores of bacterial spot in fruits.

The plant materials with the best performance were selected and will continue in the breeding program on disease resistance at the University of Brasilia.

\section{References}

Alvares, C. A., Stape, J. L., Sentelhas, P. C., Gonçalves, J. L. M., \& Sparovek, G. (2013). Köppen's climate classification map for Brazil. Meteorologische Zeitschrift, 22(6), 711-728. https://doi.org/10.1127/ 0941-2948/2013/0507

Bergamin Filho, A., Lopes, D. B., Amorim, L., Godoy, C. V., \& Berger, R. D. (1995). Avaliação de danos causados por doenças de plantas. Revisão Anual de Patologia de Plantas, 3, 133-170.

Campbell, C. L., \& Madden, L. V. (1990). Introduction to plant disease epidemiology. New York, NY: John Wiley.

Carvalho, F. I. F., Lorencetti, C., \& Benin, G. (2004). Estimativas e implicações da correlação no melhoramento vegetal. Pelotas, RS: Ed. Universitária da UFPel.

Chagas, K., Alexandre, R.S., Schmildt, E. R., Bruckner, C. H., \& Faleiro, F. G. (2016). Divergência genética em genótipos de maracujazeiro azedo, com base em características físicas e químicas dos frutos. Revista Ciência Agronômica, 47(3), 524-531. https://doi.org/10.5935/1806-6690.20160063

Costa, A. P., Nogueira, I., Peixoto, J. R., \& Blum, L. E. B. (2020). Screening of Sour Passion Fruit for Reaction to Bacterial Spot and Passion Fruit Woodiness Disease. Journal of Agricultural Science, 12(2);130-137. https://doi.org/10.5539/jas.v12n2p130

Costa, A. P., Nogueira, I., Peixoto, J. R., Vilela, M. S., Blum, L. E. B., \& Vendrame, W. (2018). Yellow passion fruit reaction to Xanthomonas axonopodis pv. passiflorae and to Cowpea aphid-borne mosaic virus. Crop Breeding and Applied Biotechnology, 18(4), 349-356. https://doi.org/10.1590/1984-70332018v18n4a53

Falconer, D. S. (1987). In M. A. Silva \& J. C. Viçosa (Trans.), Introdução à genética quantitative. Brazil: Imprensa Universitária.

Faleiro, F. G., \& Junqueira, N. T. V. (2011). Recursos genéticos: Conservação, caracterização e uso. In F. G. Faleiro, S. R. M. Andrade, \& F. B. Reis Júnior (Eds.), Biotecnologia: Estado da arte e aplicações na agropecuária (pp. 513-551). Planaltina, Brazil: Embrapa Cerrados.

Franco, M. M., \& Takatsu, A. (2004). Sensibilidade de Xanthomonas axonopodis pv. passiflorae a cobre. Bioscience Journal, 20, 207-210.

Fuhrmann, E., Junqueira, N. T. V., Blum, L. E. B., Braga, M. W., Bellon, G., \& Junqueira, K. P. (2014). Reação de híbridos interespecíficos de Passiflora spp. à Xanthomonas axonopodis pv. passiflorae. Ciência Rural, 44(8), 1404-1410. https://doi.org/10.1590/0103-8478cr20121092

Gonçalves, I. M. P. (2011). Produtividade e reação de genótipos de maracujazeiro azedo a doenças em campo e casa de vegetação (Master's thesis, University of Brasilia, DF, Brazil). Retrieved from https://repositorio.unb.br/handle/10482/9592

IBGE (Instituto Brasileiro de Geografia e Estatística). (2019). Produção Agrícola Municipal. Brasília, DF. Retrieved from https://sidra.ibge.gov.br/tabela/1613\#resultado

INMET (Instituto Nacional de Meteorologia). (2019). Gráficos. Brasília, DF. Retrieved from http://www.inmet.gov.br/portal/index.php?r=tempo/graficos 
Junqueira, N. T. V., Anjos, J. R. N., Silva, A. P. O., Chaves, R. C., \& Gomes, A. C. (2003). Reação as doenças e produtividade de onze cultivares de maracujá-azedo cultivados sem agrotóxicos. Pesquisa Agropecuária Brasileira, 38(8), 1005-1010. https://doi.org/10.1590/S0100-204X2003000800014

Junqueira, N. T. V., Sussel, A. A. B., Junqueira, K. P., Zacaroni, A. B., \& Braga, M. F. (2016). Doenças. In F. G. Faleiro, \& N. T. V. Junqueira (Eds.), Maracujá: O produtor pergunta, a Embrapa responde (pp. 169-180). Brasilia, Brazil: Embrapa Cerrados.

Kado, C. L., \& Heskett, M. S. (1970). Selective media for isolation of Agrobacterium, Corynebacterium, Erwinia, Pseudomonas and Xanthomonas. Phytopathology, 60, 969-976. https://doi.org/10.1094/ Phyto-60-969

Laranjeira, F. F. (2005). Problemas e perspectivas da avaliacão de doencas como suporte ao melhoramento do maracujazeiro. In F. G. Faleiro, N. T. V. Junqueira, \& M. F. Braga (Eds.), Maracujá germoplasma e melhoramento genético (pp. 161-183). Brasiilia, Brazil: Embrapa Cerrados.

Miranda, H. A. (2004). Incidência e severidade de Xanthomonas axonopodis pv. passiflorae, Colletotrichum gloeosporioides, Septoria passiflorae, Cladosporium herbarum e passion fruit woodiness virus em genótipos de maracujazeiro azedo cultivados no Distrito Federal (Master's thesis, University of Brasilia, DF, Brazil).

Rocha, M. R. D. (2014). Estratégias de seleção no melhoramento genético do maracujazeiro azedo (Master's thesis, Federal University of Viçosa, MG, Brazil). Retrieved from https://www.locus.ufv.br/handle/ $123456789 / 2040$

Viana, C. A. S., Pires, M. C., Peixoto, J. R., Junqueira, N. T. V., \& Blum, L. E. B. (2014). Genótipos de maracujazeiro-azedo com resistência à bacteriose. Bioscience Journal, 30, 591-598.

Zaccheo, P. V. C., Aguiar, R. S., Stendel, N. M. C., Sera, T., \& Neves, C. S. V. J. (2012). Produção e caracterização qualitativas dos frutos de híbridos de maracujazeiro-amarelo. Revista Brasileira de Fruticultura, 34(4), 1113-1120. https://doi.org/10.1590/S0100-29452012000400019

\section{Copyrights}

Copyright for this article is retained by the author(s), with first publication rights granted to the journal.

This is an open-access article distributed under the terms and conditions of the Creative Commons Attribution license (http://creativecommons.org/licenses/by/4.0/). 